\title{
EFECTIVIDAD DE LA HIGIENE ORAL PERIOPERATORIA SISTEMÁTICA EN LA REDUCCIÓN DE LAS INFECCIONES DEL TRACTO RESPIRATORIO POSTOPERATORIAS TRAS UNA CIRUGÍA TORÁCICA ELECTIVA EN ADULTOS: UNA REVISIÓN SISTEMÁTICA
}

Alexandra Gimeno Cardells

Enfermera. Hospital Universitari i Politècnic La Fe (Valencia). 


\section{RESUMEN}

\section{Introducción}

Las infecciones nosocomiales contribuyen significativamente a la morbilidad y mortalidad de los pacientes sometidos a cirugía. Las infecciones nosocomiales aumentan significativamente la duración de la estancia hospitalaria y los costes totales del hospital. Se sabe que la cirugía torácica, la ventilación mecánica y / o el ingreso en una unidad de cuidados intensivos aumentan el riesgo de infecciones respiratorias nosocomiales.

\section{Objetivo}

Esta revisión se centra en identificar, evaluar y sintetizar la mejor evidencia disponible sobre la efectividad de la higiene oral sistemática perioperatoria en la reducción de infecciones respiratorias postoperatorias en pacientes adultos sometidos a cirugía torácica no urgente.

\section{Métodos, fuentes de datos}

\section{CRITERIOS DE INCLUSIÓN}

Tipos de participantes: Pacientes mayores de 18 años que habían ingresado para cirugía torácica no urgente, independientemente de su sexo, origen étnico, gravedad del diagnóstico, comorbilidad o tratamiento previo.

Tipos de intervenciones: Higiene oral sistemática durante el perioperatorio realizada por los propios pacientes o por el personal cuidador (incluido enfermeras):

- Cepillado dental: se incluyeron los pacientes que siguieron un protocolo particular para su cepillado dental hasta siete días antes del procedimiento quirúrgico. Se consideraron los estudios si especificaban la frecuencia diaria del cepillado, el uso de un método específico para cepillarse los dientes o el uso de una pasta dental específica para el procedimiento.

- Enjuague bucal: Se incluyeron estudios si los pacientes siguieron una intervención específica de enjuague bucal (por ejemplo, enjuague orofaríngeo que contiene gluconato de clorhexidina $[0,12 \%]$ ); la solución orofaríngea (10 $\mathrm{ml}$ ) se usó como enjuague bucal, se aplicó durante 30 segundos al menos dos veces al día; y la intervención comenzó al menos un día antes de la cirugía y continuó hasta un día después de la cirugía o el alta de la UCl. Si el paciente no pudo seguir el protocolo de forma autónoma, un miembro del equipo médico realizó la higiene por el paciente.

Tipos de estudios: Ensayos controlados aleatorios y estudios cuasiexperimentales. 
Tipos de resultados: Infecciones nosocomiales respiratorias (que aparecieron durante la hospitalización o hasta 48 horas tras el alta hospitalaria) e infecciones de la herida quirúrgica.

\section{ESTRATEGIA DE BUSQUEDA}

Se realizaron búsquedas en varias bases de datos (PubMed, CINAHL, Embase, Scopus, Swemed +, Health Technology Assessment Database y Turning Research Into Practice [TRIP]) desde 1980 hasta diciembre de 2014. Se incluyeron estudios publicados en inglés, alemán, danés y sueco.

\section{CALIDAD METODOLÓGICA y EXTRACCIÓN DE DATOS}

Dos revisores independientes utilizaron la herramienta de evaluación crítica estándar del Instituto Joanna Briggs para evaluar la calidad metodológica de los estudios. Del mismo modo, el proceso de extracción de datos fue realizado independientemente por estos dos revisores utilizando herramientas del Instituto Joanna Briggs.

\section{SÍNTESIS DE LOS DATOS}

\section{Resultados}

Esta revisión incluyó seis estudios: tres ensayos clínicos aleatorizados y tres estudios cuasiexperimentales.

Los resultados cuantitativos se sintetizaron en tres metaanálisis. El primer metaanálisis, tuvo como objetivo mostrar el tamaño del efecto con respecto a la reducción de las infecciones nosocomiales, siendo el riesgo relativo (RR) 0,65 (intervalo de confianza [IC] del 95\%: 0,55-0,78).

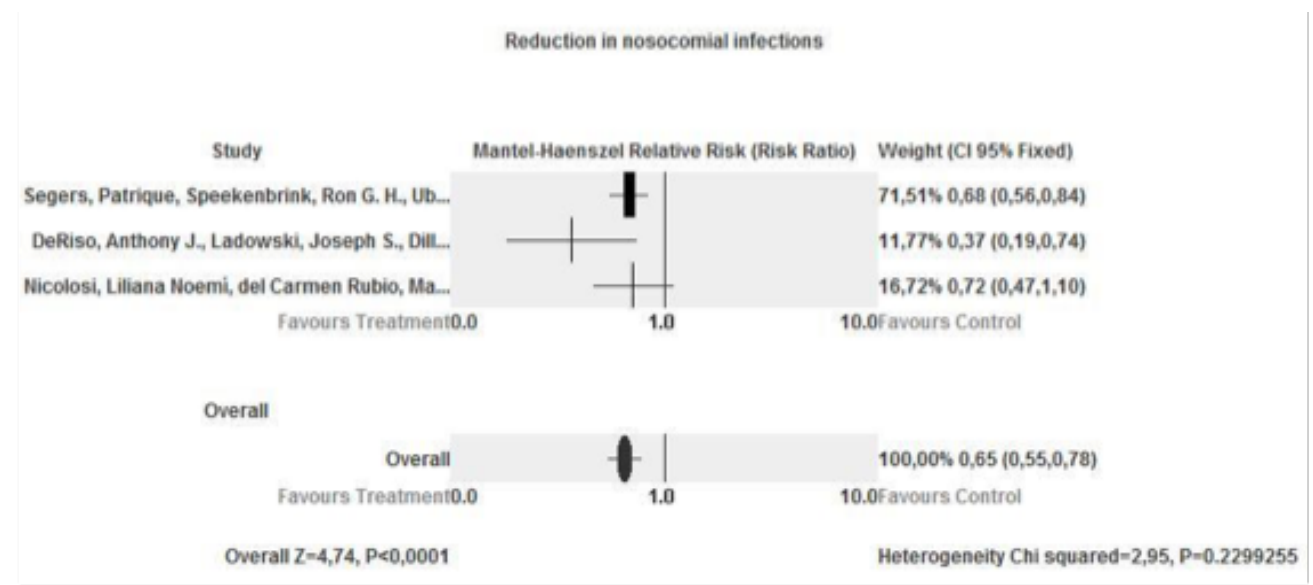

El segundo metaanálisis incluyo 4 estudios y mostró un tamaño del efecto RR 0,48 (IC del 95\%: 0,36-0,65) para infecciones del tracto respiratorio. 


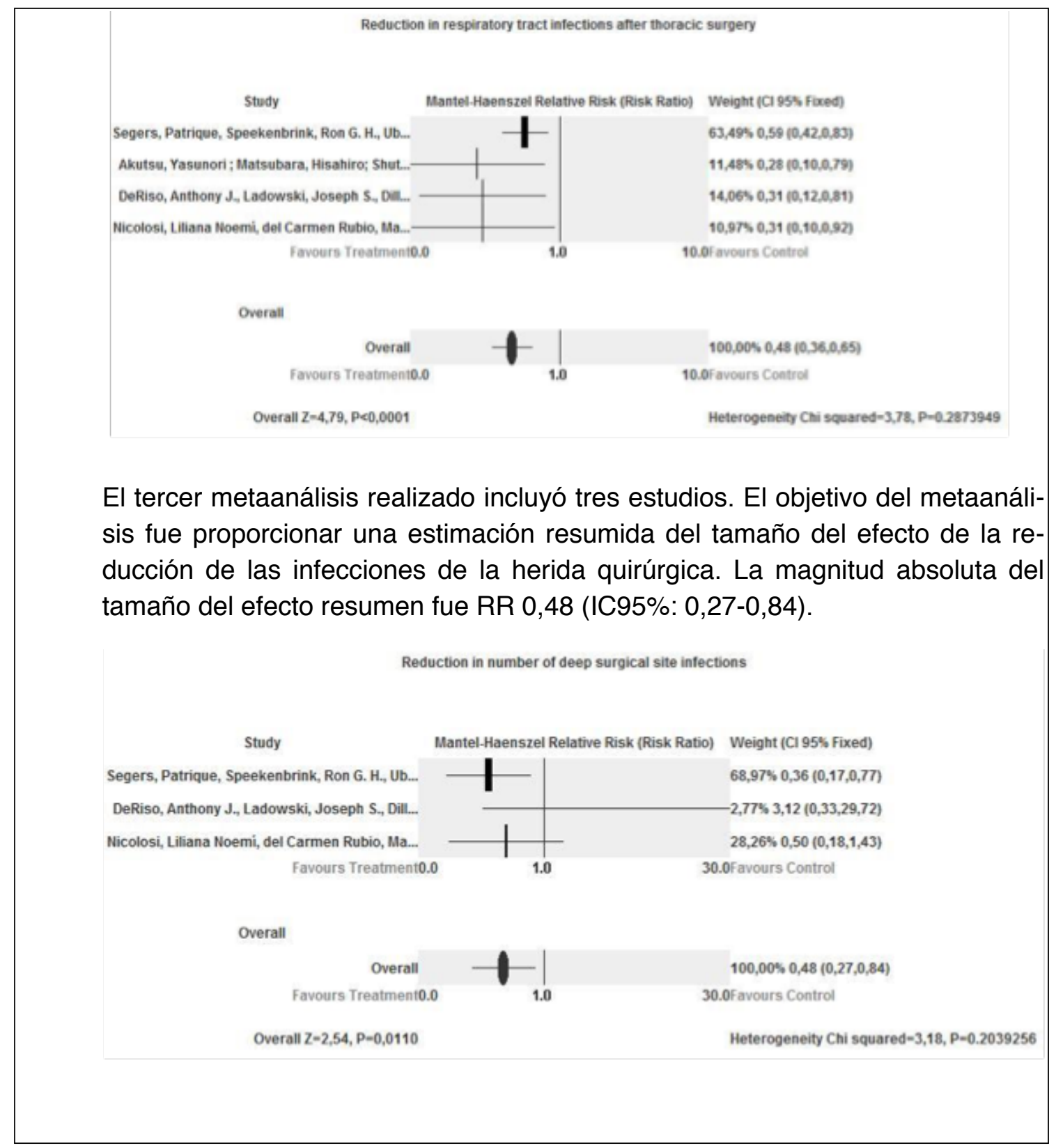




\section{RECOMENDACIONES}

La descontaminación perioperatoria de la nasofaringe y/o la orofaringe es una estrategia efectiva. La intervención es barata y puede ser realizada fácilmente por los propios pacientes. Por lo tanto, Se recomienda a los pacientes adultos sometidos a cirugía torácica no urgente que realicen, durante el perioperatorio, higiene oral sistemática con el fin de reducir las infecciones postoperatorias. (Grado A).

\section{ComentARIO}

Las infecciones nosocomiales son un factor importante en el aumento de la morbimortalidad de los pacientes. La tasa de infección nosocomial después de una cirugía cardíaca ronda el $20 \%(1,2)$ y en concreto, las infecciones nosocomiales del tracto respiratorio representan más del $50 \%$ de las infecciones nosocomiales en este grupo de pacientes (1), lo que aumenta la necesidad de antibiótico, recuperación prolongada y mayores costes económicos.

La aspiración de organismos de la orofaringe generalmente se considera el mecanismo más importante que provoca la neumonía nosocomial (3), siendo la flora del paciente la principal fuente de microorganismos (4). Los patógenos en la placa dental son factores de riesgo conocidos para la neumonía postoperatoria y la limpieza mecánica de dientes y encías juega un papel importante en la prevención de la formación de ésta ${ }^{(5)}$.

Los resultados de la revisión muestran que la descontaminación de la orofaringe a través de medidas básicas de higiene bucodental es una intervención barata y fácil de realizar en pacientes que tiene un importante efecto, la disminución de las infecciones nosocomiales.

En este sentido, las enfermeras jugamos un papel fundamental en la educación y consejo de medidas sanitarias. Aunque la recomendación de la revisión sistemática no diferencia si la actividad la realiza la enfermera u otro profesional, la higiene bucodental forma parte de los cuidados básicos responsabilidad de los profesionales enfermeros. Por lo tanto, debe formar parte de nuestra recomendaciones de cuidados en los pacientes que van a ser sometidos a una cirugía y a lo sumo, a la población general.

Respecto a la calidad de la revisión sistemática, cabe destacar que los estudios incluidos finalmente fueron 6 , concretamente 3 ensayos clínicos y 3 estudios cuasiexperimentales. Tanto las herramientas como el análisis de los datos fueron fiables y apropiados. En los resultados, la revisión sistemática muestra los resultados por separado de cada uno de los estudios incluidos y posteriormente exponen los resultados del metaanálisis. 
En cuanto a las limitaciones de la revisión, cabe destacar que el meta análisis se realizó con 3-4 de los 6 estudios incluidos en la revisión ya que en dos estudios se compararon dos ingredientes activos para enjuagues bucales o no se informaron datos relevantes. El metaanálisis con el modelo de efectos fijos se realizó en tres de los cuatro estudios incluidos. Cabe destacar que el estudio de Segers et al (5), tuvo una ponderación del $61-73 \%$ respectivamente en el metaanálisis, lo cual puede afectar la capacidad de emitir un juicio apropiado sobre la fiabilidad de los resultados obtenidos en el metaanálisis.

De cara al futuro, harían falta estudios y revisiones que tengan en cuenta el impacto económico de la implementación de este tipo de intervenciones, además de investigar la opinión del paciente sobre el significado y la idoneidad de las intervenciones.

Implementar las intervenciones en una práctica clínica será un desafío. Los pacientes necesitan obtener información sobre la intervención y estar dispuestos a asumir la responsabilidad de la descontaminación de la orofaringe antes de la admisión. Como recomendación, se debería implementar en las consultas preoperatorias instrucciones precisas sobre la higiene oral y durante el ingreso del paciente se debería supervisar y/o realizar actividades de limpieza e higiene de la orofaringe. Las recomendaciones aportadas son de grado $\mathrm{A}$, por lo que está comprobada su efectividad en la reducción de infecciones nosocomiales, y por tanto, indicada su aplicación.

\section{REFERENCIAS}

1. De Santo LS, Bancone C, Santarpino G, Romano G, De Feo M, Scardone M, et al. Microbiologically documented nosocomial infections after cardiac surgery: an 18-month prospective tertiary care centre report. Eur J Cardio Thorac Surg.2008;33(4):666-672.

2. Segers P, Speekenbrink RGH, Ubbink DT, van Ogtrop ML, de Mol BA. Prevention of Nosocomial Infection in Cardiac Surgery by Decontamination of the Nasopharynx and Oropharynx With Chlorhexidine Gluconate. JAMA. 2006;296(20):2460-2466.

3. Houston S, Hougland P, Anderson JJ, LaRocco M. Effectiveness of $0.12 \%$ chlorhexidine gluconate oral rinse in reducing prevalence of nosocomial pneumonia in patients undergoing heart surgery. Am J Crit Care. 2002;11(6):567-567-70.

4. Mojon P. Oral Health and Respiratory Infection. J Can Dent Assoc. 2002;68(6):340-5.

5. El-Solh AA, Pietrantoni C, Bhat A, Okada M, Zambon J, Aquilina A, et al. Colonization of Dental Plaques. Chest. 2004;126(5):1575-1582. 